\title{
LIPOPROTEINAS DE BAJA DENSIDAD (LDL) Y VITAMINA E
}

Lidia Cruz Neyral

\section{RESUMEN}

Los hábitos alimenticios pueden incrementar los niveles de las lipoproteínas plasmáticas principalmente las lipoproteínas de baja densidad (LDL) que son las que conducen el colesterol del hígado al tejido periférico. La peroxidación de los lípidos de la LDL es un paso importante en la aterogenicidad de esta partícula lipídica y la reducción de la concentración endógena de vitamina E contenida en la LDL acelera su peroxidación. El objetivo del presente estudio es informar y discutir la suplementación de Vitamina E como protector de la LDL oxidada.

Palabras claves: Lipoproteìna de baja densidad (LDL), Vitamina E, peroxidación.

\section{SUMMARY}

The eating habits can increase the levels of plasma lipoprotein, particularly low density lipoproteins (LDL), that transport cholesterol from liver to peripheral tissue. Lipid peroxidation of LDL is an important step in the atherogenicity and a reduced endogenous concentration of vitamin E contained in LDL accelerates the peroxidation. The aim of this study is to report and discuss the supplementation of vitamin E, as a protector of oxidized LDL.

Key words: Low-density-lipoprotein (LDL), Vitamin E, Peroxidation.

\section{LIPOPROTEINAS PLASMATICAS}

La primera causa de muerte en el Perú, así como en los países desarrollados son las enfermedades cardiovasculares, atribuible a varios factores, entre ellos a las alteraciones de los niveles de las lipoproteínas plasmáticas y trastornos del metabolismo de los lípidos (Heinecke etal, 1998).

Las lipoproteínas son partículas esféricas, de un tamaño menor que los hematíes y solo son visibles al microscopio electrónico; compuestos por lípidos (colesterol, esteres de colesterol, triglicéridos y fosfolípidos) y proteínas (apolipoproteínas). Las lipoproteínas se clasifican de acuerdo a su densidad (Cuadro 1) en Quilomicrones (QM), lipoproteína de muy baja densidad (VLDL), lipoproteína de baja densidad (LDL) y lipoproteína de alta densidad, HDL (Herrera, 1993).
Los QM son las lipoproteínas de mayor tamaño y su función es transportar triglicéridos (TG), el colesterol y otros componentes liposolubles de la dieta desde el intestino al hígado. Las VLDL son lipoproteínas secretadas a nivel hepático, que contiene colesterol, fosfolípidos, triglicérido, Apo B-100 y pequeñas cantidades de Apo E y Apo C. Presentan cierta similitud con los QM, aunque son de menor tamaño. Los remanentes de VLDL, ricos en esteres de colesterol se conocen como lipoproteína de densidad intermedia (IDL).

Las LDL se generan en el plasma, proceden de la IDL y contienen sólo una molécula de Apo B-100. El papel de las LDL es transportar el colesterol a los tejidos periféricos y regular la síntesis de novo del colesterol en estos lugares. Está ampliamente descrito que concentraciones elevadas de LDL en el plasma constituyen un factor de riesgo (STEINBERG et al., 1989). 
Cuadro 1: Lipoproteínas plasmáticas

\begin{tabular}{|c|c|c|c|c|c|}
\hline & Quilomicrones & VLDL & IDL & LDL & HDL \\
\hline $\begin{array}{l}\text { Densidad } \\
(\mathrm{g} / \mathrm{ml})\end{array}$ & $<0,95$ & $0,95-1,006$ & $1,006-1,019$ & $1,019-1,063$ & $1,063-1,210$ \\
\hline $\begin{array}{l}\text { Movilidad } \\
\text { Electroforética }\end{array}$ & Origen & Pre- beta & $\begin{array}{l}\text { Entre beta y } \\
\text { pre - beta }\end{array}$ & Beta & Alfa \\
\hline $\begin{array}{l}\text { Diámetro } \\
(\mathrm{nm})\end{array}$ & $>70$ & $25-70$ & $22-24$ & $19-23$ & $4-10$ \\
\hline $\begin{array}{l}\text { Peso molecular } \\
\text { (daltons) }\end{array}$ & $\begin{array}{c}0,4-30 \\
\times 10^{9} \\
\end{array}$ & $\begin{array}{l}5-10 \\
\times 10^{6} \\
\end{array}$ & $\begin{array}{r}4-5 \\
\times 10^{6} \\
\end{array}$ & $\begin{array}{c}1,8-2,8 \\
\times 10^{6} \\
\end{array}$ & $\begin{array}{c}1,8-3,6 \\
\times 10^{5} \\
\end{array}$ \\
\hline $\begin{array}{l}\text { Relación } \\
\text { Lípido/ Proteína }\end{array}$ & $99: 1$ & $90: 10$ & $85: 15$ & $80: 20$ & $50: 50$ \\
\hline $\begin{array}{l}\text { Lípidos más } \\
\text { Abundantes }\end{array}$ & $\begin{array}{c}\text { TG } \\
\text { Exógenos }\end{array}$ & $\begin{array}{c}\text { TG endógenos } \\
\text { y CE }\end{array}$ & TG endógenos & CE y FC & PL y CE \\
\hline $\begin{array}{l}\text { Principales } \\
\text { Apoproteínas }\end{array}$ & $\begin{array}{l}\text { A-I, B-48, } \\
\text { C-I,C-II, CIII }\end{array}$ & $\begin{array}{l}\text { B-100, C-I, } \\
\text { C-II,C-III,E }\end{array}$ & B- $100, E$ & B-100 & A-I, A-II \\
\hline
\end{tabular}

TG: triaglicéridos; CE: ésteres de colesterol; FC: colesterol libre; PL: fosfolípidos

LIPOPROTEÍNA DE BAJA DENSIDAD (LDL)

LDL humana se define como la población de lipoproteínas que pueden ser aisladas por ultracentrifugación en gradiente de densidad con un rango de de 1.019 a $1.063 \mathrm{gr} / \mathrm{ml}$. LDL tiene un peso molecular de 1.8 a 2.8 millones de daltons, si tomamos un valor promedio de su peso molecular de 2.5 millones, cada partícula tendría aproximadamente 1600 moléculas de ésteres de colesterol y 170 moléculas de triglicéridos, que juntos forman un core central lipofílico (Herrera, 1993).

Este core central se encuentra rodeado por una monocapa formada principalmente por unas 700 moléculas de fosfolípidos (particularmente fosfatidilcolina) y 600 moléculas de colesterol libre. La región polar de los fosfolípidos se encuentran en la superficie de LDL contribuyendo a su solubilidad en la fase acuosa. El número total de moléculas de ácidos grasos unidos a las diferentes clases de lípidos de la LDL es en promedio de 2700. De estos, alrededor del $50 \%$ son ácidos grasos poliinsaturados, principalmente ácido linoleico (COSGROVE etal., 1987).

El contenido de ácidos grasos y su patrón de distribución varía considerablemente de persona en persona probablemente a los diversos hábitos dietéticos. La variación en el contenido de ácidos grasos poliinsaturados y la relación saturados/poliinsaturados tiene un efecto significativo sobre el comportamiento oxidativo de las distintas partículas de LDL ya que estos ácidos grasos son más susceptibles de oxidación por mecanismos no enzimáticos (Trostchansky et al, 2001).

LDL contiene la apolipoproteína B-100 (apo B-100). La apo B-100 es una proteína constituida por 4536 aminoácidos y un peso de $512 \mathrm{kDa}$. De su secuencia aminoacídica se destacan la presencia de triptofanos, tirosinas y metioninas $(37,152$ y $78 \mathrm{~mol} / \mathrm{mol}$ respectivamente) así como cuatro residuos de cisteína libres.

Las LDL también transportan diversos antioxidantes como componentes estructurales. Entre ellos se destaca el $\alpha$-tocoferol con una concentración en $\mathrm{nmol} / \mathrm{mg}$ de LDL que equivale a aproximadamente seis moléculas de $\alpha$-tocoferol por partícula de LDL. Los demás antioxidantes, como son el $\gamma$-tocoferol, $\beta$-caroteno,- $y$ ubiquinol-10 que se encuentran en cantidades menores (Herrera, 1993).

\section{ESTRÉS OXIDATIVO}

Durante las actividades normales de la célula, varios procesos en su interior pueden producir especies reactivas del oxígeno, conocidas como ROS (reactive oxygen species). Las más comunes ROS son el peróxido de hidrógeno $\left(\mathrm{H}_{2} \mathrm{O}_{2}\right)$, el ión superoxido $\mathrm{O}_{2}^{-} \mathrm{y}$ el radical hidroxilo $\left(\mathrm{OH}^{\circ}\right)$. Estos componentes cuando se presentan en altas concentraciones pueden dañar las proteínas, lípidos y el ácido desoxirribonucleico $(\mathrm{ADN})$. Los antioxidantes pueden prevenir las concentraciones altas de ROS y prevenir el daño celular. Los antioxidantes celulares pueden ser enzimáticos (catalasa, glutationa peroxidasa, superóxido dismutasa) o no enzimático (glutationa, tioles, algunas vitaminas, metales o fotoquímicos como isoflavonas, polifenoles y flavonoides). 
Los ROS juegan un papel importante en los procesos fisiológicos normales que incluyen respuestas a factores de crecimiento, respuesta inmune y la apoptosis de células dañadas; sin embargo una producción incrementada puede contribuir a desarrollar enfermedades cardiovasculares, sobre todo en dietas que no contienen una adecuada cantidad de antioxidantes (Trostchansky et al 2003).

La lipoperoxidación es el proceso en el cual el oxígeno molecular es incorporado a moléculas de lípidos insaturados para formar hidroperóxidos lipídicos $(\mathrm{LOOH})$. El proceso de ataque y daño oxidativo que sufren los lípidos insaturados se debe a reacciones en cadena mediadas por radicales libres, iniciadas por la abstracción de un átomo de hidrógeno del metileno bisalílico del lípido insaturado por un radical libre reactivo y seguido por una secuencia de reacciones propagadoras.

La lipoperoxidación ocurre en los ácidos grasos insaturados (PUFAs) formándose los radicales peroxilos (LOO). El radical peroxilo es un importante intermediario en la cadena de propagación porque una vez formado, continuará la cadena de reacciones oxidativas abstrayendo un átomo de hidrógeno de otros grupos alquilo cercanos. Este ciclo de reacciones propagadoras se repite a través de la abstracción de hidrógenos y formación de $\mathrm{LOO}^{-}$, siempre que se encuentran disponibles suficientes moléculas de $\mathrm{O}_{2} \mathrm{y}$ sustratos lipídicos insaturados (Ozer \& Azzi, 2000)

\section{LDLOXIDADA}

La hipótesis oxidativa de la aterosclerosis sostenida por diversos investigadores (Steinbrecher et al, 1984; Parthasarathy et al 1985; Esterbauer et al, 1989; Witztum, 1995) postula que las placas de ateroma se forman a partir de células (principalmente macrófagos y musculares lisas) cargadas con lípidos (ésteres de colesterol), como consecuencia de modificaciones oxidativas de la LDL (LDL oxidada u ox-LDL). Las LDL son internalizadas en las células a través del receptor normal de LDL (receptor apoE/apoB) por endocitosis mediada por receptor. Este es un proceso que impide la entrada excesiva de lipoproteínas a las células; además su regulación está coordinada con la del metabolismo del colesterol impidiendo la acumulación nociva de este lípido. A diferencia de la LDL nativa, la ox-LDL es reconocida por receptores barrenderos o "scavenger" a nivel de las células endoteliales y musculares lisas, monocitos y macrófagos por un proceso no regulado. Mientras la LDL es oxidada, la lipoproteína pierde su habilidad de ser reconocida por el receptor de la LDL incrementándose a su vez la afinidad por el receptor scavenger.
Durante la oxidación de la LDL a una forma reconocida por los receptores scavenger, existe una disminución de la cantidad de grupos $\varepsilon$-amino libres de los residuos de lisina de las LDL lo cual explica el cambio en la especificidad del receptor. La acumulación intracelular de lípidos, combinada con la disminución de la degradación de los lípidos oxidados, resulta en la conversión de los macrófagos en células espumosas formándose la estría grasa que constituye una acumulación focal de lípidos en la íntima arterial y que representa la lesión más precoz del proceso (Steinberg, et al, 1989).

La hipercolesterolemia produce daño endotelial, pero los mecanismos que causan esta injuria no están completamente aclarados. Probablemente el endotelio, células musculares lisas, neutrófilos, monocitos y plaquetas puedan ser la fuente de radicales libres (RL), los cuales generarían el estrés oxidativo (Witztum, 1995; Salonen et al, 1997; Huynn, 1999, Trostchansky et al, 2001).

El daño oxidativo que se produce por el desequilibrio entre fenómenos antioxidantes/proxidantes parece crucial en el origen de la ateroesclerosis, y esto aumenta la posibilidad que los antioxidantes como la vitamina $\mathrm{C}$, betacaroteno y en especial el alfatocoferol (vitamina E) puedan prevenir o retardar el desarrollo de esta enfermedad (Miller et al, 1998).

\section{VITAMINA E COMO ANTIOXIDANTE DE LDL}

El estrés oxidativo y su asociación con trastornos cardiovasculares y neurológicos, ha desviado la atención a un conjunto de sustancias a las cuales anteriormente no se les reconocía su importancia. La vitamina $\mathrm{E}$ es una de ellas, considerándose como un factor protector en estas enfermedades por sus acciones fundamentales como agente antioxidante y en el mantenimiento de la integridad de las membranas.

La vitamina E pertenece al grupo de vitaminas liposolubles ampliamente distribuida en los alimentos. $\mathrm{Su}$ principal función descrita es como antioxidante natural que reacciona con los radicales libres solubles en los lípidos de las membranas, también desempeña una función fisicoquímica en el ordenamiento de las membranas lipídicas, estabilizando las estructuras de membranas. Su absorción es relativamente pobre y va unida a los lípidos de la dieta. Se ha descrito neuropatía que comienzan a partir del daño en la membrana axonal y decursa hacia una axonopatía distal que afecta las fibras mielinizadas de gran calibre.

Las deficiencias de vitamina $\mathrm{E}$ son particularmente importantes en los recién nacidos prematuros por sus deficientes reservas corporales y deterioro de la absor- 
ción de las grasas. La mayoría de las secuelas secundarias a la deficiencia de vitamina E son subclínicas, se han estudiado afectaciones en el sistema inmunológico relacionado con la deficiencia de la vitamina $\mathrm{E}$ en la proliferación celular y su función fagocítica. En las enfermedades crónicas no transmisibles y, particularmente, las cardiovasculares y el cáncer, se han estudiado las relaciones entre las vitaminas antioxidantes y sus fisiopatologías, no existiendo resultados consistentes y las fuerzas de asociación encontradas no son elevadas, ya que existen muchos factores de riesgo que son comunes a ambas enfermedades y modifican su aparición (DIEZ et al., 1997, ESTER bauer et al., 1996).

Esta vitamina E constituye el mejor sistema de neutralización de radicales libres generados en medios lipídicos, siendo muy efectiva para prevenir la lesión causada por los RL en las membranas celulares o en las lipoproteínas (Bonet et al, 1998).

Estudios recientes sugieren que la génesis de la lesión ateromatosa de la pared arterial involucra la incorporación de la LDL oxidada a los monocitosmacrófagos arteriales, que se convierten así en células espumosas, núcleo fundamental del ateroma (Bonet et al, 1998; Bernedo, 1998). Secundariamente proliferan otros tipos celulares, especialmente células musculares lisas, originando la placa de ateroma madura.

Datos reportados por Witzum (1995) demuestran que un suplemento adecuado de vitamina E aumenta su contenido en la LDL, protegiéndola frente a la oxidación. Sin embargo, en el ser humano se requiere suplementos de vitamina E de 1-2 g/día para saturar a la LDL y así disminuir la susceptibilidad a la peroxidación lipídica.

Esta última hipótesis estaría apoyada por los resultados obtenidos en numerosas investigaciones, ya que ambos grupos que recibieron un suplemento de vitamina $\mathrm{E}$ (con dieta hipercolestero-lémica y con dieta normal), muestran marcada disminución de la lipoperoxidación ( $p<0,005$ ), comparado con los grupos que no recibieron suplemento de vitamina E. Por lo tanto, el suplemento de vitamina $E$ puede favorecer la capacidad antioxidante en el suero y proteger contra la lipoperoxidación in vivo.

El nivel de triglicéridos es crucial en la modulación del comportamiento metabólico de las lipoproteínas; así, una hipertrigliceridemia supone un incremento en los niveles de lipoproteínas ricas en triglicéridos (VLDL y quilomicrones), lo cual tiene una profunda repercusión en la cantidad, calidad y en la función de las lipoproteínas transportadoras de colesterol (LDL y HDL).

En el ser humano se ha encontrado actividad de una proteína transferidora de colesterol (CETP) que promueve el intercambio de ésteres de colesterol y triglicéridos entre los diferentes tipos de lipoproteínas, de tal manera que capta los ésteres de colesterol de la HDL y los entrega a cambio de triglicéridos a las lipoproteínas ricas en triglicéridos (VLDL y quilomicrones). Estas HDL que recibieron triglicéridos adquieren menor tamaño y menor densidad, disminuyendo su vida media, por lo que son rápidamente retiradas de la circulación (Can et al, 1998).

La Vitamina E puede ayudar a prevenir o retrasar enfermedades coronarias (Traber, 2007; Hope, 2000) Los investigadores ha detectado que cambios oxidativos en el colesterol LDL (llamado frecuentemente colesterol "malo") promueve las obstrucciones (arterosclerosis) en las arterias coronarias que pueden conducir a los ataques del corazón. La vitamina $\mathrm{E}$ puede ayudar a prevenir o retrasar enfermedad cardíaca coronaria limitando la oxidación del LDL-colesterol (Sen et al. 2006).

La vitamina $\mathrm{E}$ también puede ayudar a prevenir la formación de los coágulos de la sangre, que podrían conducir a un ataque del corazón. Los estudios de observación han asociado índices más bajos de la enfermedad cardíaca a la ingesta más alta de vitamina E. Un estudio de aproximadamente 90.000 enfermeras sugirió que la incidencia de la enfermedad cardíaca fuera el $30 \%$ a el $40 \%$ más baja entre enfermeras con ingesta más alta de vitamina $\mathrm{E}$ en la dieta y en suplementos. Los investigadores encontraron que la ventaja evidente fue asociada principalmente a la toma de vitamina $E$ en suplementos dietéticos. La ingesta de la vitamina $\mathrm{E}$ en alimentos no fue asociada a la reducción significativa del riesgo cardiaco (Dietrich et al, 2006).

Una revisión en 1994 de 5.133 hombres y mujeres finlandeses de entre 30-69 años también sugirió que la ingestión dietética creciente de la vitamina $\mathrm{E}$ estaba asociada a una mortalidad disminuida de la enfermedad cardíaca (Verhagen et al, 2006).

El efecto protector de la vitamina $\mathrm{E}$ en el desarrollo de la aterosclerosis (Ozer \& Azzi, 2000 Dietrich et al., 2006) también ha sido demostrado en conejos, los que recibieron una dieta hipercolesterolémica $(2 \%)$ más vitamina $\mathrm{E}(50 \mathrm{mg} / \mathrm{Kg})$, encontrándose una prevención en la formación de lesiones ateroscleróticas en la aorta.

Se ha reportado que la suplementación con vitamina $\mathrm{E}$ ayuda a reducir los niveles de colesterol de LDL hasta un $17 \%$ y el estudio más reciente realizado por Yeun et al, 2011 indicaron que individuos con niveles elevados de LDL fueron sometidos a una dieta suplementada con vitamina $\mathrm{E}$ durante cuatro meses evidenciándose una reducción del $11 \%$ y aquellos suplementados durante 
seis meses disminuyeron sus niveles de LDL en un $17.5 \%$.

Landrier et al. 2010 señalaron que la vitamina $\mathrm{E}$ ayuda a prevenir enfermedades del corazón a través de la reducción de los niveles de LDL, al afectarlas tasas metabólicas de colesterol en el intestino. Los investigadores encontraron que la vitamina $\mathrm{E}$ ayuda a regular los genes que están involucrados en la síntesis del colesterol, reduciendo eficazmente los niveles de LDL.

Aunque estas observaciones son prometedoras, algunos otros estudios se cuestionan la eficacia de los suplementos de la vitamina $\mathrm{E}$ en la prevención de la enfermedad cardíaca. El estudio The Heart Outcomes Prevention Evaluation (HOPE) monitoreo a casi 10.000 pacientes durante 4,5 años que estaban bajo alto riesgo de un ataque al corazón (Institute of Medicine, 2000). En este estudio de la intervención los individuos que recibieron diariamente magnesio 265 (400 IU) con vitamina $\mathrm{E}$ no experimentaron perceptiblemente diferencias en sus comportamientos cardiovasculares comparados con los recibieron un placebo (píldora del azúcar). Los investigadores sugirieron que era inverosímil que el suplemento de la vitamina $\mathrm{E}$ proporcionara alguna protección contra enfermedad cardiovascular en el estudio HOPE citado. No obstante, este estudio continua, con la meta de determinar si una duración más larga de la intervención con suplementos de la vitamina E proporcionará alguna protección contra enfermedad cardiovascular (Yeun et al., 2011).

\section{LITERATURA CITADA}

BERNEDO L.1998. Intervienen los triglicéridos en la patogenia de la ateroesclerosis. Caduceus. 1(3):814.

BONET Serra B, Viana M. Arribas, E. Herrera C. 1998. Efectos teratogénicos de la diabetes. Papel de los radicales libres. Endocrinología. 45:292-7.

CAN Bruce R, Chovinard A, Tall AR. 1998.Plasma lipid transfer. Protein, high-density lipoproteins, and reverse cholesterol transport. Annu Rev Nutr; 18:297-330.

COSGROVE JP, Church DF, Pryor WA. 1987.The kinetics of the autoxidation of polyunsaturated fatty acids. Lipids 22:299-304.

DIETRICH M, Traber MG, Jacques PF, Cross CE, Hu Y, Block G. 2006. Does $\gamma$-tocopherol play a role in the primary prevention of heart disease and cancer? Areview. Am J Coll Nutr. 25:292-9.

DIEZ MN, Frei B, Vita JA, Keaney Jr JF 1997. Antioxidants and atherosclerotic heart disease. N Engl J Med 1;7:408-14.

ESTERBAUER H, Gebicki J, Puhl H, Jurgens G. 1992. The role of lipid peroxidation and antioxidants in oxidative modification of LDL. Free Radic Biol Med. 13:341-90.
ESTERBAUER, H., Rotheneder, M., Striegl, G., Waeg, G., Ashy, A., Sattler, W. and Jurgens, G. 1989. Vitamin E and other lipophilic antioxidants protect LDL against oxidation. Fat Sci. Technol. 91:316-324.

HEINECKE JW. 1998. Oxidants and antioxidants in the pathogenesis of atherosclerosis: implications for the oxidized low density lipoprotein hypothesis. Atherosclerosis .141(1):1-15.

HERRERA E. 1993. Metabolismo de las Lipoproteínas. In: Graw-Hill IM, ed. Elementos de Bioquímica.

HILTUNER T, Luoma J, Nikkari T, Yla-Herttuala S.1988. Expression of LDL receptor, VLDL receptor, LDL receptor-related protein and scavenger receptor in rabbit atherosclerotic lesions. Circulation 97:1079-86.

HOPE. 2000. The heart outcomes prevention evaluation study investigators. Vitamin E supplementation and cardiovascular events in high-risk patients. New Engl. J. Med. 342:154160.

HUYNN L. 1999. Combination therapy of cholesterol reduction and L-arginine supplementation controls accelerated vein graft atheroma. Ann Vasc Surg 13:484-93.

INSTITUTE OF MEDICINE. Food and Nutrition Board. 2000. Dietary Reference Intakes: Vitamin C, Vitamin E, Selenium, and Carotenoids. Washington, DC: National Academy Press.

LANDRIER, J. Gourantion, E.; Reboul, E. Cardinault, N, El Yazidi, C. 2010. Vitamin E decreases endogenous cholesterol synthesis and apo AI mediated cholesterol secretion in caco-2 cells. Journal of Nutritional Biochemistry. 21 (12): 1207-1213.

MILLER ER, Appel LJ, Risby TH. 1998. Effect of dietary patterns on measures of lipid peroxidation. Circulation. 2390-5.

OZER NK, Azzi A. 2000. Effect of vitamin E on the development of atherosclerosis. Toxicology. 148(2-3):179-85.

PARTHASARATHY, S., Steinbrecher, U., Barnett, J., Witztum, J. and Steinberg, D.1985. Essential role of phospholipase A2 activity in endothelial cell induce modification of low density lipoprotein. Proc. Natl. Acad. Sci. 82: 3000-3004.

SALONEN JT, Nyyssonen K, Salonen R, Porkkatas Arotoho E, Toumainan T, Diezfalusy V, Björkhem I. 1997. Lipoprotein oxidation and progression of carotid atherosclerosis. Circulation. 5:840-4.

SEN CK, Khanna S, Roy S. 2006.Tocotrienols: vitamin E beyond tocopherols. Life Sci;78:208898

STEINBERG, D., Parthasarathy, S., Carew, T., Khoo, J. and Witztum, J. 1989. Modifications of low density lipoprotein that increase its atherogenicity. New Engl. J. Med. 320: 915-924. 
STEINBRECHER, U., Parthasarathy, S., Leake, D., Witztum, J. and Steinberg, D. 1984. Modification of low-density lipoprotein by endothelial cells involves lipid peroxidation and degradation of low-density lipoprotein phospholipids. Proc. Natl. Acad. Sci. 83:3883-3887.

TRABER MG. 2007. Vitamin E regulatory mechanisms. Annu Rev Nutr 27:347-62.

TRABER MG. Vitamin E. In: Shils ME, Shike M, Ross AC, Caballero B, Cousins R, eds. 2006. Modern Nutrition in Health and Disease. 10th ed. Baltimore, MD: Lippincott Williams \& Wilkins, 396-411.

TrOSTCHANSKY, A., Batthyany, C., Botti, H., Radi, R., Denicola, A., and Rubbo, H. 2001. Formation of lipid-protein adducts in low-density lipoprotein by fluxes of peroxynitrite and its inhibition by nitric oxide. Arch. Biochem. Biophys. 395: 225-232.

TROSTCHANSKY, A., Ferrer-Sueta, G., Batthyany, C., Botti, H., Batinic-Haberle, I., Radi, R., and
Rubbo, H. 2003. Peroxynitrite-mediated LDL oxidation is inhibited by manganese porphyris in the presence of uric acid. Free. Rad. Biol. Med. 35: 1293-1300.

U.S. Department of Agriculture, Agricultural Research Service. 2010. USDA National Nutrient Database for Standard Reference, Release 23

VERHAGEN H, Buijsse B, Jansen E, Bueno-deMesquita B. 2006. The state of antioxidant affairs. Nutr Today. 41:244-50.

WESTHUYZEN J. 1997. The oxidation hypothesis of atherosclerosis: an update. Ann Clin Lab Sci 1997; 27:1-10.

WITZTUM JL. 1995 La oxidación como hipótesis de la ateroesclerosis. Lancet [Ed. en Español]. 26(2):113-6.

YEUN, K. H; Wong, J.W.; Lim, A.B.; Ng, B.H.; Chong, W. P. 2011. Effect of mixed- tocotrienols in hypercholesterolemic subjects. Functional Foods in health and disease. 3, 106-117. 